\title{
Design procedure of robust QFT-based controller for continuous-flow grain dryer plant.
}

\begin{abstract}
Quantitative Feedback Theory (QFT) is a well known robust controller that deals with plant uncertainty. QFT has been applied to many industrial applications, however it never been applied to any types of grain dryer plant. Grain dryer plant prone to parameter uncertainty and needs a robust controller in order to maintain a good quality of product output. The objective of this paper is to explain step-by-step design procedure of QFT design for a continuous-flow grain dryer plant. The designed QFT-based controller is also tested and compared with PID controller via simulation. The test results showed that the QFT-based controller works better than PID controller in terms of shorter settling time and smaller percentage of overshoot for the grain dryer plant under study and at the same time insensitive to parameter changes i.e. input and output disturbances.
\end{abstract}

Keyword: Disturbance; Grain dryer; PID; Quantitative feedback theory; Robust control; Uncertainty 